WU-AP $/ 43 / 94$

hep-th/9408084

\title{
Superradiance around Rotating Dilatonic Black Holes
}

\author{
Jun-ICHIROU KogA and KeI-ICHI MAEDA ${ }^{(a)}$ \\ Department of Physics, Waseda University, Shinjuku-ku, Tokyo 169, Japan
}

\begin{abstract}
We consider a superradiance effect around rotating dilatonic black holes. We analyze two cases: one is an exact solution with the coupling constant $\alpha=\sqrt{3}$, which effective action is reduced from the 5-dimensional Kaluza-Klein theory, and the other is a slowly rotating dilatonic black holes with arbitrary coupling constant. We find that there exists a critical value $(\alpha \sim 1)$, which is predicted from a superstring model, and the superradiant emission rate with coupling larger than the critical value becomes much higher than the Kerr-Newman case $(\alpha=0)$ in the maximally charged limit. Consequently, 4-dimensional primordial black holes in higher dimensional unified theories are either rotating but almost neutral or charged but effectively non-rotating.
\end{abstract}

July, 1994

(a) electronic mail : maeda@cfi.waseda.ac.jp 


\section{Introduction}

In unified theories of fundamental interactions, a dilaton field usually appears and couples to other known matter field. It may play some important role in the history of the early universe or in astrophysics. It may also provide us a window to see some evidence of higherdimensional unification. The coupling constant of the dilaton field depends on the dimension of an internal space and a unification theory. Therefore it is important to see an effect of the coupling on observable phenomena and to investigate a dependence of the coupling constant.

In the Einstein-Maxwell-dilaton theory, which action is described as

$$
S=\frac{1}{16 \pi} \int d^{4} x \sqrt{-g}\left[R-2(\nabla \phi)^{2}-e^{-2 \alpha \phi} F^{2}\right]
$$

electrically (or magnetically) charged non-rotating black hole[1] shows very interesting thermodynamical properties depending on a coupling constant $\alpha$ of a dilaton field $\phi$. (We adopt the units of $c=\hbar=G=1$.) Remind that the case of $\alpha=0$ corresponds to the EinsteinMaxwell theory, in which the Reissner-Nordström solution is a unique spherically symmetric black hole. If $\alpha<1$, the thermodynamical property is similar to the Reissner-Nordström black hole, namely, its Hawking temperature vanishes in the limit of extremely charged hole. When we consider a superstring theory $(\alpha=1)$, its temperature in the extreme limit becomes non-zero and finite, while it diverges in the case of $\alpha>1$. The last case includes the 5-dimensional Kaluza-Klein theory, where $\alpha=\sqrt{3}$. This property of dilatonic black holes indicates that the Hawking radiation may be drastically affected by the coupling constant.

Shiraishi 2] also discussed a discharge process by the superradiant modes for non-rotating dilatonic black holes. Although he could not present a definite conclusion because of his analytic WKB approach, his analysis suggests that there is a critical value of the coupling constant. Below the critical value, the superradiant effect is similar to that of the ReissnerNordström black hole, while it changes qualitatively beyond that value, i.e., as the coupling constant increases, the discharge proceeds very rapidly. 
In this letter, we study the rotating black holes and investigate the effect of dilaton field on the superradiance. We also look for whether there is a critical value beyond which the property changes qualitatively.

One difficulty in our study is that we do not know the exact solutions of rotating black holes except for two cases: $\alpha=0$ (Kerr-Newman) and $\alpha=\sqrt{3}$. The latter is the 4 dimensional rotating black hole solution in the 5-dimensional Kaluza-Klein theory derived by Frolov et al [3]. We call it the Kaluza-Klein black hole. We have, however, the approximate solutions of slowly rotating black holes with arbitrary coupling constant, which were found by Horne and Horowitz [4], and by Shiraishi[ [0].

Here, we use these two exact solutions and the approximate solutions to analyze superradiance around rotating dilatonic black holes. We assume a charge of a black hole is conserved (it is true for a central charge), and then consider only a massless neutral scalar particle.

\section{Superradiance around Kaluza-Klein Black Hole}

Before showing the calculations of superradiance and spontaneous emission rate, we have to mention the rotating Kaluza-Klein black hole solution[3]. It is given by

$$
\begin{aligned}
d s^{2}= & -\frac{\Delta-a^{2} \sin ^{2} \theta}{B \Sigma} d t^{2}-2 a \sin ^{2} \theta \frac{1}{\sqrt{1-v^{2}}} \frac{Z}{B} d t d \varphi \\
& +\left[B\left(r^{2}+a^{2}\right)+a^{2} \sin ^{2} \theta \frac{Z}{B}\right] \sin ^{2} \theta d \varphi^{2}+\frac{B \Sigma}{\Delta} d r^{2}+B \Sigma d \theta^{2} \\
A_{t}= & \frac{v}{2\left(1-v^{2}\right)} \frac{Z}{B^{2}}, \quad A_{\varphi}=-a \sin ^{2} \theta \frac{v}{2 \sqrt{1-v^{2}}} \frac{Z}{B^{2}}, \quad \phi=-\frac{\sqrt{3}}{2} \ln B
\end{aligned}
$$

where, $\Delta, \Sigma, Z$ and $B$ are functions of $r$ and $\theta$, and given by,

$$
\Delta=r^{2}-2 \mu r+a^{2}, \quad \Sigma=r^{2}+a^{2} \cos ^{2} \theta, \quad Z=\frac{2 \mu r}{\Sigma}, \quad B=\left(1+\frac{v^{2} Z}{1-v^{2}}\right)^{\frac{1}{2}} .
$$

The mass $M$, the electric charge $Q$ and the angular momentum $J$ of black hole are obtained by the parameters $v(|v|<1), \mu$ and $a$ as,

$$
M=\mu\left[1+\frac{v^{2}}{2\left(1-v^{2}\right)}\right], \quad Q=\frac{\mu v}{1-v^{2}}, \quad J=\frac{\mu a}{\sqrt{1-v^{2}}} .
$$


Here we assume $J$ is positive without loss of generality. The horizon radius $r_{H}$ is given by,

$$
r_{H}=\mu+\sqrt{\mu^{2}-a^{2}}, \quad\left(\mu^{2} \geq a^{2}\right)
$$

This black hole has three independent global hairs, that is, the mass $M$, the charge $Q$ and the angular momentum $J$. When $Q=0$, it reduces to the Kerr black hole, while the solution with $J=0$ corresponds to the non-rotating dilatonic black hole [1] with the coupling constant $\alpha=\sqrt{3}$. The condition of $\mu^{2} \geq a^{2}$ for the horizon to exist is rewritten as

$$
\left(\frac{J}{M^{2}}\right)^{2} \leq \frac{1}{4}\left[2-10\left(\frac{Q}{M}\right)^{2}-\left(\frac{Q}{M}\right)^{4}+2\left(1+2\left(\frac{Q}{M}\right)^{2}\right)^{3 / 2}\right] .
$$

(see Fig. 1). It should be noticed here that the solution with $|Q|=2 M$ (and $J=0$ ) is not a black hole solution because a naked singularity appears, as is pointed out in [1, 6].

The angular velocity $\Omega_{H}$ of this black hole is

$$
\Omega_{H}=\frac{a \sqrt{1-v^{2}}}{r_{H}^{2}+a^{2}},
$$

which shows an interesting feature near $|Q|=2 M$, similar to that of the black-hole temperature[1]. When $J=0$, obviously $\Omega_{H}$ vanishes. While, if $J \neq 0, \Omega_{H} \rightarrow \infty$ in the limit of $|Q| \rightarrow 2 M$ $(J \rightarrow 0)$. It is discontinuous at $|Q|=2 M$. Although $|Q|=2 M$ is not the black hole solution, the solution with $|Q| \lesssim 2 M$ represents a rapidly spinning black hole.

Now we discuss a superradiance. We consider a massless neutral scalar field $\Phi$, described by the Klein-Gordon equation

$$
\square \Phi=0
$$

In the background fields of the rotating Kaluza-Klein black hole, we can separate variables. The spheroidal equation is written in the same form as the Kerr-Newman case, setting

$$
\Phi=\frac{\chi(r)}{R(r)} S(\theta) e^{i m \varphi} e^{-i \omega t}
$$


where

$$
\left.R^{2}(r) \equiv B \Sigma\right|_{\theta=0}=\left(r^{2}+a^{2}\right)\left(1+\frac{v^{2}}{1-v^{2}} \frac{2 \mu r}{r^{2}+a^{2}}\right)^{\frac{1}{2}} .
$$

We perform a coordinate transformation from $r$ to $r^{*}$

$$
d r^{*}=\frac{R^{2}(r)}{\Delta(r)} d r
$$

to get the radial equation in a usual form as

$$
\left[\frac{d^{2}}{d r^{* 2}}+\left(\omega^{2}-m \Omega(r)\right)^{2}-V^{2}(r)\right] \chi\left(r^{*}\right)=0
$$

where

$$
\begin{aligned}
\Omega(r) & \equiv \frac{a}{\sqrt{1-v^{2}}} \frac{2 \mu r}{R^{4}(r)} \\
V^{2}(r) & \equiv \frac{\Delta(r)}{R^{2}(r)}\left\{\frac{\lambda}{R^{2}(r)}+\frac{1}{R(r)} \frac{d}{d r}\left[\frac{\Delta(r)}{R^{2}(r)} \frac{d R(r)}{d r}\right]-\frac{m^{2} a^{2}}{R^{6}(r)}\left[r^{2}+a^{2}+\frac{2 \mu r}{1-v^{2}}\right]\right\}
\end{aligned}
$$

The eigenvalue $\lambda$ of the spheroidal equation for the angular function $S(\theta)$ is obtained perturbatively [7] as

$$
\lambda=l(l+1)+\lambda_{2}(l, m) a^{2} \omega^{2}+\lambda_{4}(l, m) a^{4} \omega^{4}+\mathcal{O}\left(a^{6} \omega^{6}\right)
$$

where $\lambda_{2}$ and $\lambda_{4}$ are some known functions of $l$ and $m$, and $\lambda_{2}(1,1)=4 / 5$ and $\lambda_{2}(1,1)=$ $-4 / 875$. To see that this expansion is sufficiently powerful to calculate $\lambda$, for example, we can point out the fact that $a \omega \leq 0.5$ for the present model with $m=1$, which equality happens in the extremely rotating case (in the Kerr limit).

From Eq. (12), we find that a superradiance occurs for $0<\omega<\Omega_{H}$. When we quantize a field, we naturally expect spontaneous emission for the superradiant modes, by which a rotating black hole loses the energy and angular momentum[B]. The rates of the spontaneous emission for a scalar field is calculated by the formulae

$$
\frac{d M}{d t}=-\frac{1}{2 \pi} \sum_{l, m} \int_{0}^{m \Omega_{H}} \omega\left(|A|^{2}-1\right) d \omega, \quad \frac{d J}{d t}=-\frac{1}{2 \pi} \sum_{l, m} \int_{0}^{m \Omega_{H}} m\left(|A|^{2}-1\right) d \omega .
$$


where $|A|^{2}$ is a reflection coefficient of scalar wave described by Eq.(12) .

In what follow, we assume the charge of the black hole is positive $(v \geq 0)$ without loss of generality. We consider a process in which a charge of the black hole is conserved. We will show our numerical results only for $l=m=1$ mode which is the most dominant.

Remind that a highly rotating case is similar to the Kerr black hole. Hence we shall restrict our analysis mainly into a highly charged case $(Q \lesssim 2 M)$. The result of the Kaluza-

Klein black hole $(\alpha=\sqrt{3})$ is shown in Fig.2 for $J=0.01 M^{2}$ with the Kerr-Newman case ( $\alpha=0)$ to see the effect of the dilaton coupling. In the Kerr-Newman case, both energy and angular momentum emission rates first increase when the charge of the black hole becomes large, and then they drop to small values near the extreme hole. On the other hand, both rates in the Kaluza-Klein black hole always increase and seem to diverge to infinity in the limit of the maximal black hole. Therefore, we may conclude that the superradiant emission is affected very much by the dilaton coupling, and the emission rates in the Kaluza-Klein black hole are much higher than the Kerr-Newman case, in particular in the extreme limit.

\section{Superradiance around Slowly Rotating Black Holes}

Next, we analyze a dependence of the emission rates on the coupling constant $\alpha$. To see this, we consider the slowly rotating solution. This solution is described as [4, [5]

$$
\begin{aligned}
d s^{2} & =-\frac{\Delta(\tilde{r})}{R^{2}(\tilde{r})} d t^{2}+\frac{R^{2}(\tilde{r})}{\Delta(\tilde{r})} d \tilde{r}^{2}+R^{2}(\tilde{r})\left(d \theta^{2}+\sin ^{2} \theta d \varphi^{2}\right)-2 a f(\tilde{r}) \sin ^{2} \theta d t d \varphi \\
A_{t} & =\frac{Q}{\tilde{r}}, \quad A_{\varphi}=-a \sin ^{2} \theta \frac{Q}{\tilde{r}}, \quad \phi=\frac{\alpha}{1+\alpha^{2}} \ln \left(1-\frac{\tilde{r}_{-}}{\tilde{r}}\right) .
\end{aligned}
$$

where we introduced new radial coordinate $\tilde{r}$ by a transformation, $r=\tilde{r}-\tilde{r}_{-}$, and

$$
\begin{aligned}
\Delta(\tilde{r}) & =\left(\tilde{r}-\tilde{r}_{+}\right)\left(\tilde{r}-\tilde{r}_{-}\right), \quad R(\tilde{r})=\tilde{r}\left(1-\frac{\tilde{r}_{-}}{\tilde{r}}\right)^{\alpha^{2} /\left(1+\alpha^{2}\right)} \\
f(\tilde{r}) & =\frac{\left(1+\alpha^{2}\right)^{2}}{\left(1-\alpha^{2}\right)\left(1-3 \alpha^{2}\right)}\left(\frac{\tilde{r}}{\tilde{r}_{-}}\right)^{2}\left(1-\frac{\tilde{r}_{-}}{\tilde{r}}\right)^{2 \alpha^{2} /\left(1+\alpha^{2}\right)} \\
& -\left(1+\frac{\left(1+\alpha^{2}\right)^{2}}{\left(1-\alpha^{2}\right)\left(1-3 \alpha^{2}\right)}\left(\frac{\tilde{r}}{\tilde{r}_{-}}\right)^{2}+\frac{1+\alpha^{2}}{\left(1-\alpha^{2}\right)}\left(\frac{\tilde{r}}{\tilde{r}_{-}}\right)-\frac{\tilde{r}_{+}}{\tilde{r}}\right)\left(1-\frac{\tilde{r}_{-}}{\tilde{r}}\right)^{\left(1-\alpha^{2}\right) /\left(1+\alpha^{2}\right)}(18)
\end{aligned}
$$


with

$$
\tilde{r}_{ \pm}=\frac{\left(1+\alpha^{2}\right)\left(M \pm \sqrt{M^{2}-\left(1-\alpha^{2}\right) Q^{2}}\right)}{\left(1 \pm \alpha^{2}\right)}, \quad a=\frac{2\left(1+\alpha^{2}\right) J}{\left(1+\alpha^{2}\right) \tilde{r}_{+}+\left(1-\alpha^{2} / 3\right) \tilde{r}_{-}}
$$

This solution is valid only when $a$ is sufficiently small. Although $f(\tilde{r})$ seems to diverge at $\alpha=1 / \sqrt{3}, \alpha=1$ and $\tilde{r}_{-}=0, f(\tilde{r})$ approaches a finite limiting value exists at each point.

The angular velocity $\Omega_{H}$ of this solution diverges for $\alpha \geq 1 / \sqrt{3}$ in the maximally charged limit, but vanishes for $\alpha<1 / \sqrt{3}$. There seems to exist a critical value of the coupling constant, beyond which we expect that the superradiant phenomena would change drastically (see §. 2). Since the maximally charged limit is no longer valid in the present approximation, $\alpha=1 / \sqrt{3}$ may not be this critical value. However, from the analysis of slowly rotating black holes we may still find some qualitative difference in the superradiant phenomena for a large coupling constant as we shall see now.

In this approximate solution, we can write down the radial part of the Klein-Gordon equation as

$$
\left[\frac{d^{2}}{d r^{*^{2}}}+(\omega-m \Omega(\tilde{r}))^{2}-V^{2}(\tilde{r})\right] \chi\left(r^{*}\right)=0
$$

where

$$
\begin{aligned}
\Omega(\tilde{r}) & \equiv \frac{a f(\tilde{r})}{R^{2}(\tilde{r})} \\
V^{2}(\tilde{r}) & \equiv \frac{\Delta(\tilde{r})}{R^{2}(\tilde{r})}\left[\frac{l(l+1)}{R^{2}(\tilde{r})}+\frac{1}{R(\tilde{r})} \frac{d}{d \tilde{r}}\left(\frac{\Delta(\tilde{r})}{R^{2}(\tilde{r})} \frac{d R(\tilde{r})}{d \tilde{r}}\right)\right]
\end{aligned}
$$

and $r^{*}$ is defined by

$$
d r^{*}=\frac{R^{2}(\tilde{r})}{\Delta(\tilde{r})} d \tilde{r}
$$

Fixing the small angular momentum $J=0.01 M^{2}$, we change the charge $Q$. We have analyzed for $\alpha=0.5,0.9,1.1,1.5$, and 2.0 in addition to the cases of $\alpha=0$, and $\sqrt{3}$, which were already analyzed in $\S .2$ but are included in the present calculations to see the accuracy of the present approximation. The results of the numerical calculations are shown in Fig.3.

This calculation shows that when we increase the coupling constant, the superradiant 
emission rates are enhanced. In particular, for sufficiently large $\alpha$, they diverge in the maximally charged limit, although they seem to approach to a finite value for the small $\alpha$ case. There seems to be a critical value of the coupling constant, below which the emission rates are similar to the Kerr-Newman case, while beyond which they diverge in the maximally charged limit. Although the present solution we used is only valid for a slowly rotating

limit, the comparison of the results with the exact solutions of $\alpha=0$, and $\sqrt{3}$ suggests that we may extrapolate the present analysis to the case of rather highly charged black hole for which one may expect the present approximation is broken down. From the figure, we find that there is a big change near $\alpha \sim 1$ in the behavior of the emission rates, which is consistent with the previous analysis in $\S .2$. We can speculate that the critical value is 1 , which is reduced from a superstring model, as in the case of black-hole temperature $\mathbb{1}$.

\section{Discussion}

Here we shall discuss an evolution of rotating dilatonic black holes created in nearly extreme states. If a black hole has small charge initially, the effect of the dilaton coupling is small because the dilatonic black hole in the limit of $Q \rightarrow 0$ always approaches the Kerr solution. On the other hand, if we consider a highly charged black hole, the black hole with larger coupling constant $\alpha$ than the critical value $\alpha \sim 1$ will lose their angular momentum much faster than the black hole with small $\alpha$. For example, the Kaluza-Klein black hole loses a half of its initial angular momentum by the factor $10^{-10}$ shorter than the Kerr-Newman does. (see Fig. 2).

As for the dependence of coupling constant, we may conclude that there is a critical value below and beyond which the qualitative behavior in the superradiance changes drastically. The critical value would be unity, which is reduced from a superstring model.

In this paper, we have considered only the case of conserved charge. If a discharge process exists, black holes with any $\alpha$ will approach quickly to the Kerr solution, as is pointed out 
in the Kerr-Newman case [10]. Shiraishi [2] also pointed out that a nonrotating black hole with larger $\alpha$ will lose its charge faster than that with small $\alpha$. Therefore we expect that total emission of energy of black hole with larger $\alpha$ is larger initially. But eventually, the difference will get much smaller after some evolution.

The contribution from the Hawking thermal radiation may become more important than the superradiant effect during the evolution, because the temperature of black hole with large $\alpha$ is greater than that with small $\alpha$ and it diverges in the maximally charged limit. If it is the case, our conclusion in this paper would be changed. As Holzhey and Wilczek [9] pointed out, however, the Hawking radiation may not be so important if the coupling constant is larger than the critical value, because the potential barrier get very large in the maximally charged limit and may prevent particles from passing through it from the horizon to infinity. This is now under investigation by numerical calculation.

\section{Acknowledgment}

We would like to thank T. Tachizawa for useful discussions. This work was supported partially by the Grant-in-Aid for Scientific Research Fund of the Ministry of Education,

Science and Culture (No. 06302021 and No. 06640412), and by Waseda University Grant for Special Research Projects.

\section{References}

[1] G. W. Gibbons and K. Maeda, Nucl. Phys. B 298 (1988) 741;

D. Garfinkle, G. T. Horowitz and A. Strominger, Phys. Rev. D 43 (1991) 3140

[2] K. Shiraishi, Mod. Phys. Lett. A 7 (1992) 3449

[3] V. Frolov, A. Zelnikov and U. Bleyer, Ann. Phys. (Leipzig) 44 (1987) 371

[4] J. H. Horne and G. T. Horowitz, Phys. Rev. D 46 (1992) 1340

[5] K. Shiraishi, Phys. Lett. A 166 (1992) 298

[6] G. W. Gibbons and D. L. Wiltshire, Ann. Phys. (N. Y.) 167 (1986) 201

[7] C. J. Bouwkamp, Phillips. Res. Rep. 5 (1950) 87

[8] W. G. Unruh, Phys. Rev. D 10 (1974) 3194 
[9] C. F. E. Holzhey and F. Wilczek, Nucl. Phys. B 380 (1992) 447

[10] D. N. Page, Phys. Rev. D 14 (1976) 3260

\section{Figure Captions}

Fig. 1: The ranges of the angular momentum $J / M^{2}$ and the charge $Q / M$ for event horizons to exist in (1) the Kerr-Newman and (2) the Kaluza-Klein black holes.

Fig. 2: Comparison of the superradiant emission rates of the Kaluza-Klein black holes with those of the Kerr-Newman black holes, fixing the angular momentum $J=$ $0.01 M^{2}$ : (1) and (2) are the emission rates of energy and angular momentum for the Kaluza-Klein black hole, respectively, while (3) and (4) are the same for the Kerr-Newman black hole.

Fig. 3: Superradiant emission rate of the energy (a) and the angular momentum (b) for a slowly rotating black holes with $(1) \alpha=0$ (the result by the exact solution is denoted by the circles), (2) $\alpha=0.5$, (3) $\alpha=0.9$, (4) $\alpha=1.1$, (5) $\alpha=1.5$, (6) $\alpha=\sqrt{3}$ (the result by the exact solution is denoted by the triangles), and (7) $\alpha=2.0$. The charge is normalized by the maximal value $Q_{\max }=\sqrt{1+\alpha^{2}} M$. 


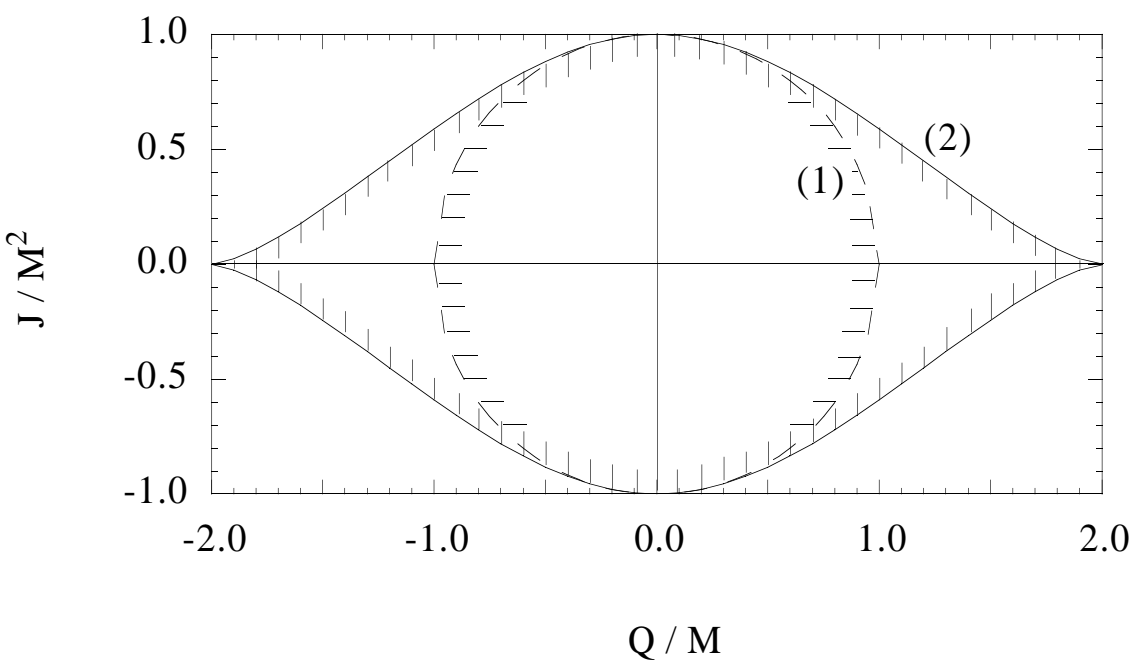

Fig.1

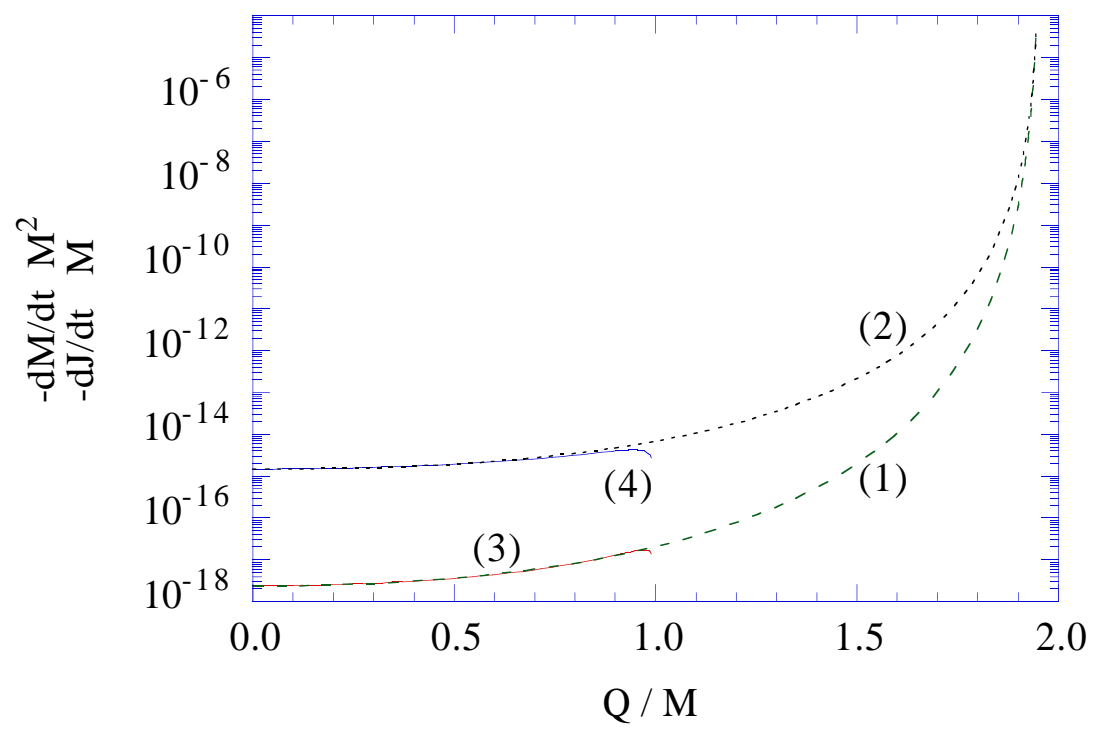

Fig. 2 


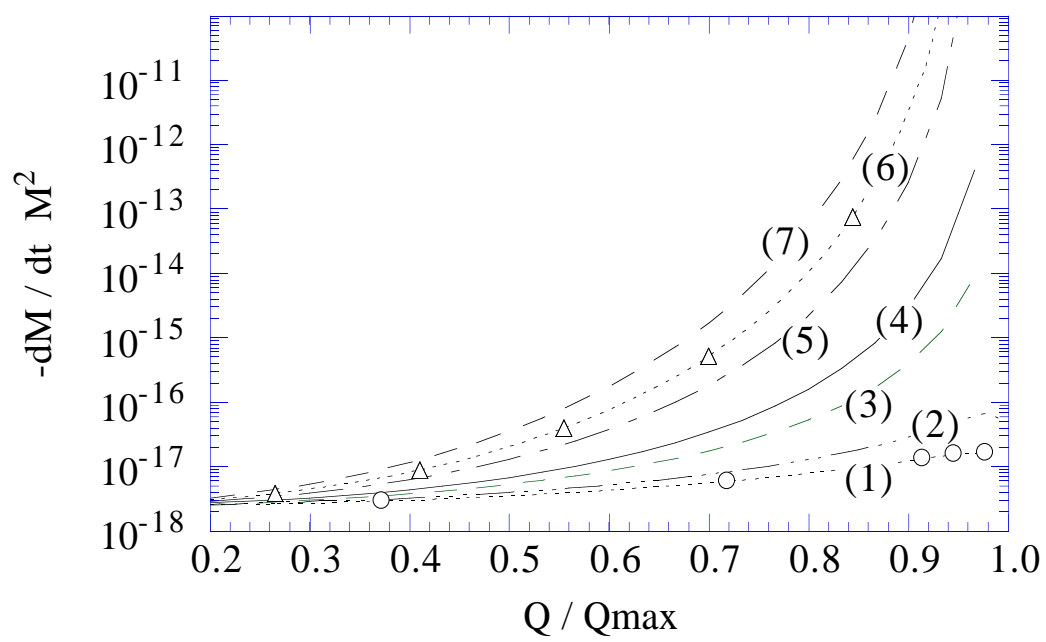

(a)

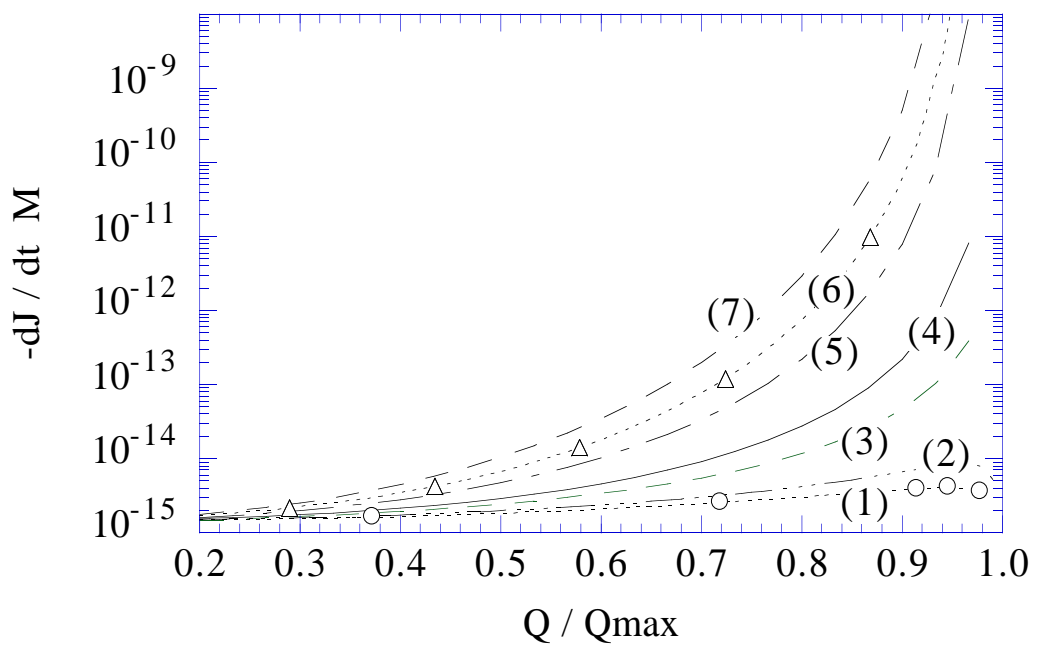

(b)

Fig.3 
This figure "fig1-1.png" is available in "png" format from: http://arxiv.org/ps/hep-th/9408084v2 
This figure "fig1-2.png" is available in "png" format from: http://arxiv.org/ps/hep-th/9408084v2 